\title{
Single-shot high-throughput phase imaging with multibeam array interferometric microscopy (MAIM)
}

Jie Dong ${ }^{1, *}$, Ali K. Yetisen ${ }^{1}$, Chen Zhao ${ }^{2}$, Xingchen Dong ${ }^{1}$, Franziska Brändle ${ }^{1}$, Qi Wang ${ }^{1}$, Martin Jakobi ${ }^{1}$, Dieter Saur ${ }^{2,3}$, and Alexander W. Koch ${ }^{1}$

${ }^{1}$ Institute for Measurement Systems and Sensor Technology, Technical University of Munich, Theresienstraße 90/N5, Munich 80333, Germany

${ }^{2}$ Institute of Translational Cancer Research and Experimental Cancer Therapy, Klinikum rechts der Isar, Technical University of Munich, Einsteinstraße 25, Munich 81675, Germany

${ }^{3}$ Division of Translational Cancer Research, German Cancer Research Center (DKFZ) and German Cancer Consortium (DKTK), Heidelberg 69120, Germany

*E-mail: jie.dong@tum.de

Supplementary figures and text:

Figure S1 FOV calibration and phase alignments for synthesizing the multiple FOV subsets.

Figure S2 Resolution estimation of MAIM via frequency analysis.

Figure S3 Resolution comparison between MAIM and the conventional DHM.

Figure S4 Quantifying the SPB of a spatial-bandwidth efficiency optimized $3 f$-MAIM.

Figure S5 Schematic of a possible implementation of dual-channel MAIM.

Figure S6 Brief description of Video S1. 


\section{Supplementary information}
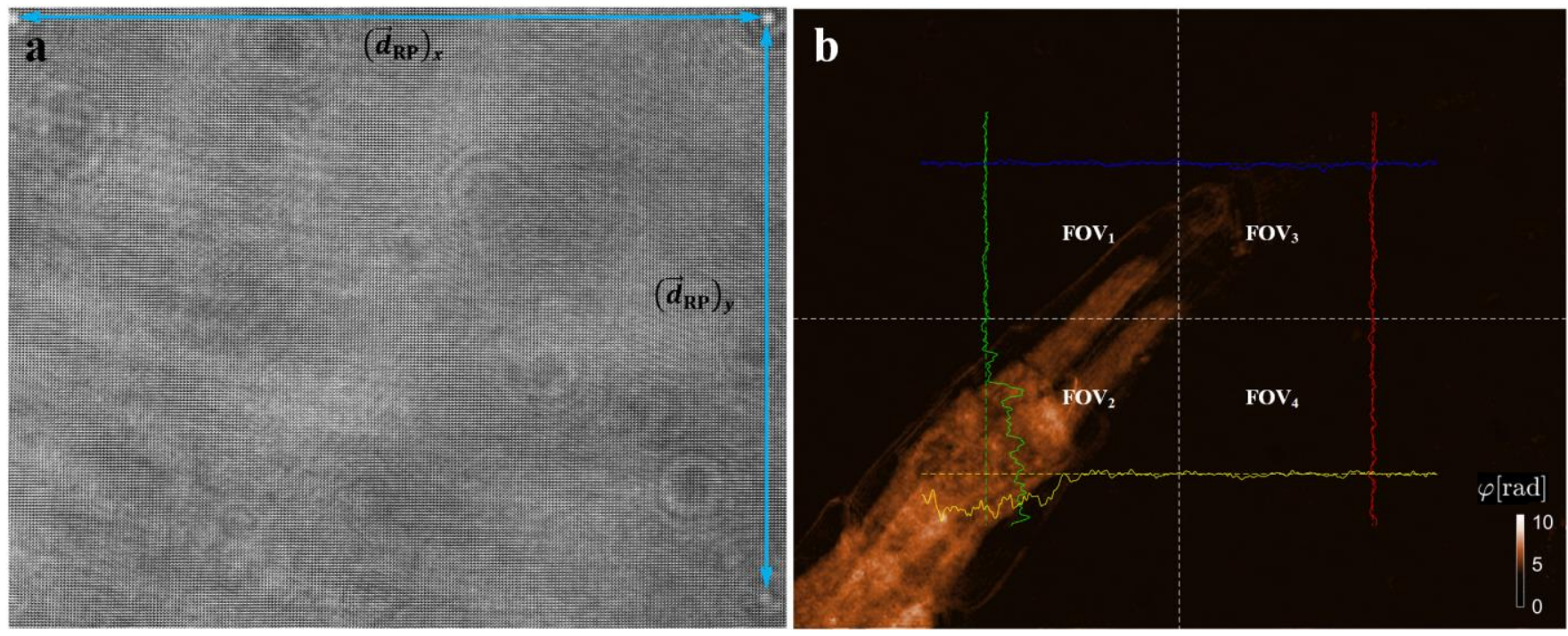

Figure S1: FOV calibration and phase alignments for synthesizing the multiple FOV subsets. (a) Spatially multiplexed interferogram for the space offset calibration of 4 -MAIM. The circle markers represent the diffraction replicas of the sample image in the recording plane, indicating the space offset amounts among the variable FOV subsets. The horizontal and the vertical components of $\vec{d}_{\mathrm{RP}}$ are constrained to match the CMOS size, ensuring no gaps or overlaps exist among the multiple FOV subsets. (b) Phase alignments for the 4 -MAIM image of a Daphnia tail. The phase image subsets are aligned by subtracting the mean values of the references (the sample-free background areas in each subset) from the respective subsets. The phase profiles spanning variable FOV subsets are continuous at the junctions, indicating that the subset phase images are well aligned. 


\section{Supplementary information}
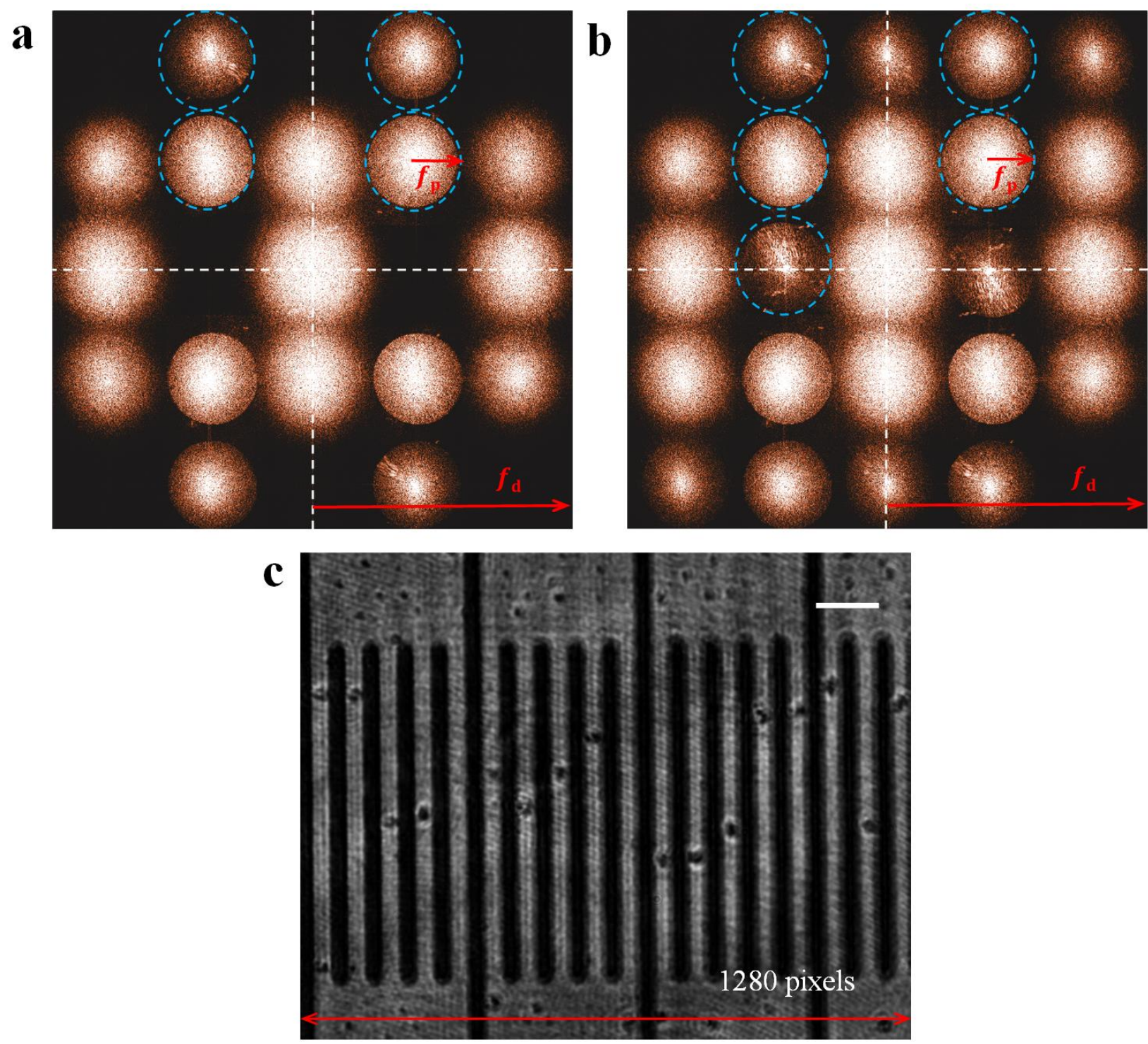

Figure S2: Resolution estimation of MAIM via frequency analysis. The FS components distribution of (a) $4 f$ MAIM and (b) $5 f$-MAIM. The maximum detectable frequency $f_{\mathrm{d}}$ is given by the sampling procedure. $f_{\mathrm{d}}$ equals $1 /\left(2 \mathrm{~S}_{\mathrm{IP}}\right)$, where $\mathrm{S}_{\mathrm{IP}}$ represents the image pixel size given by the magnification factor. $f_{\mathrm{p}}$, the maximum frequency limited by the pupil function of the MAIM imaging system. (c) Calibration of $\mathrm{S}_{\text {IP }}$ with a calibration slide. Scale bar, $20 \mu \mathrm{m}$.

The resolution of the current setup can be estimated using the frequency analysis method. A biological tissue sample was adopted for demonstration. As the sample has high-frequency microstructures that exceed the resolution limit of the objective lens, the circle shape of its FS components clearly shows the perfect pupil function of the current imaging system (Figure S2 a and b). Besides, a customized 2D spatial filter with rectangle apertures was adopted 


\section{Supplementary information}

to mark the pupil function of the spatial filter for multibeam array interference. Due to the perfect circle shape of the FS components, which is given by the imaging system, it can be concluded that the rectangular pupil function given by our customized spatial filters exceeds that of the resultant pupil function. In addition, the resolution of the current imaging system can be estimated by calibrating $\mathrm{S}_{\mathrm{IP}}$ (Figure $\mathrm{S} 2 \mathrm{c}$ ). $f_{\mathrm{p}}$ equals $\sim 1 / 6 \cdot f_{\mathrm{d}}$, corresponding to a resolution of $1 / f_{\mathrm{p}} \approx 1.64 \mu \mathrm{m}$. In the case of coherent illumination $(632 \mathrm{~nm})$, where the illumination is parallel to the optical axis, the period of the smallest features that can be observed with the microscope is given by $\lambda / \mathrm{NA}_{\mathrm{obj}} \approx 1.58$ $\mu \mathrm{m}$, which is close to $1 / f_{\mathrm{p}}$. 


\section{Supplementary information}
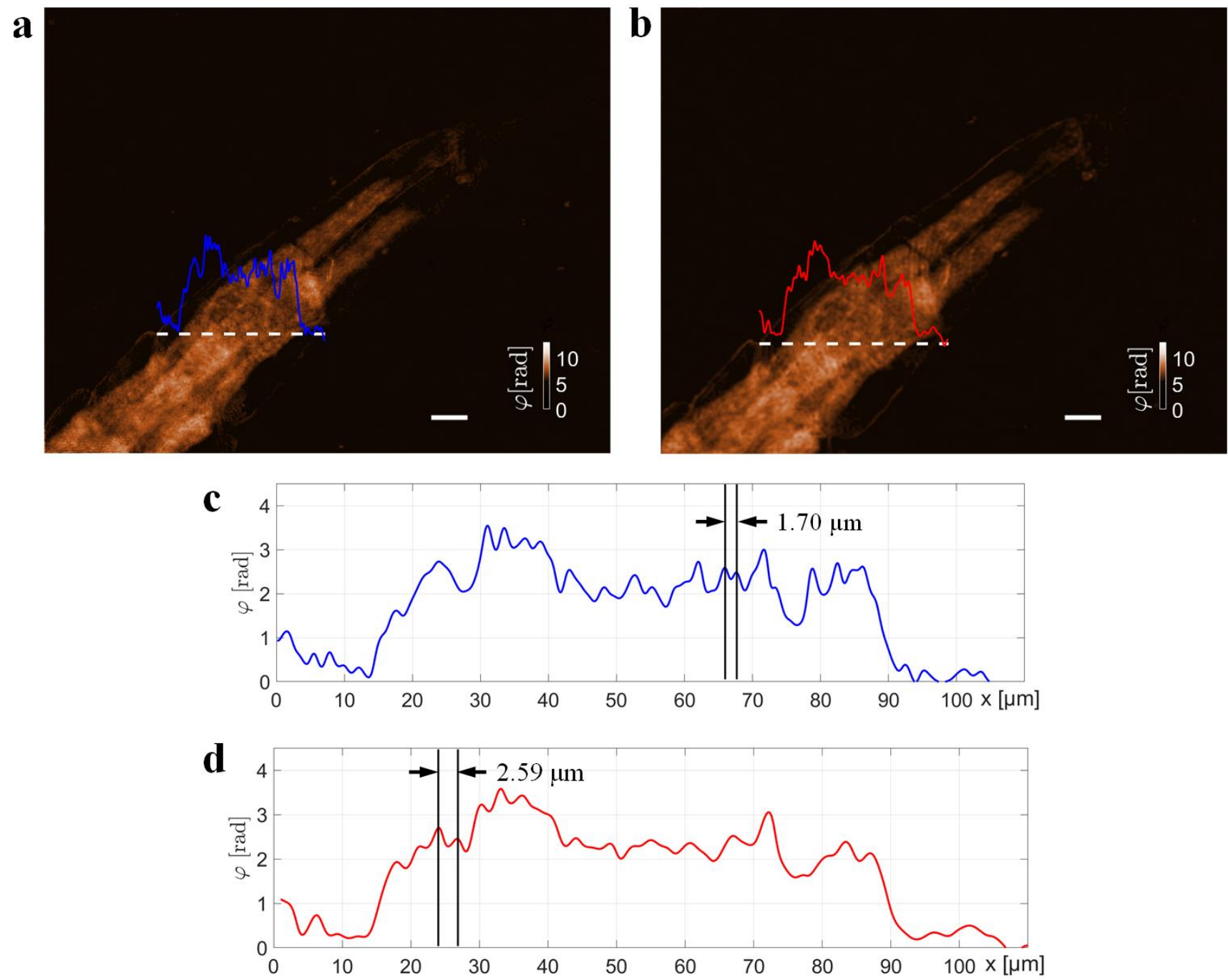

Figure S3: Resolution comparison between MAIM and the conventional DHM. The phase images of a Daphnia tail obtained by using (a) 4f-MAIM with a 20x objective lens (0.4NA) and (b) the conventional DHM with a 10x objective lens (0.25NA). (c), (d) Phase profiles along the dotted lines in (a), (b), respectively. The smallest observable sizes are $\sim 1.70 \mu \mathrm{m}$ and $\sim 2.59 \mu \mathrm{m}$, respectively. Scale bars, $20 \mu \mathrm{m}$.

We also experimentally observed the corresponding resolution limits. Although the two phase images (Figure S3 a, b) show almost the same FOV, the image of $4 f$-MAIM presents more details due to its higher NA. The smallest observable sizes provided by MAIM and the conventional DHM approach the corresponding theoretical resolution limits under coherent illumination $\left(\lambda / \mathrm{NA}_{\mathrm{obj}}\right), \sim 1.58 \mu \mathrm{m}$ and $\sim 2.53 \mu \mathrm{m}$, respectively (Figure $\mathrm{S} 3 \mathrm{c}, \mathrm{d}$ ).

Since these two images have the same FOV but the image of MAIM provides a higher resolution, it's also proved that MAIM increases the SBP of the conventional DHM by a factor of $\sim\left(\mathrm{NA}_{\mathrm{MAIM}} / \mathrm{NA}_{\mathrm{DHM}}\right)^{2}$. 


\section{Supplementary information}
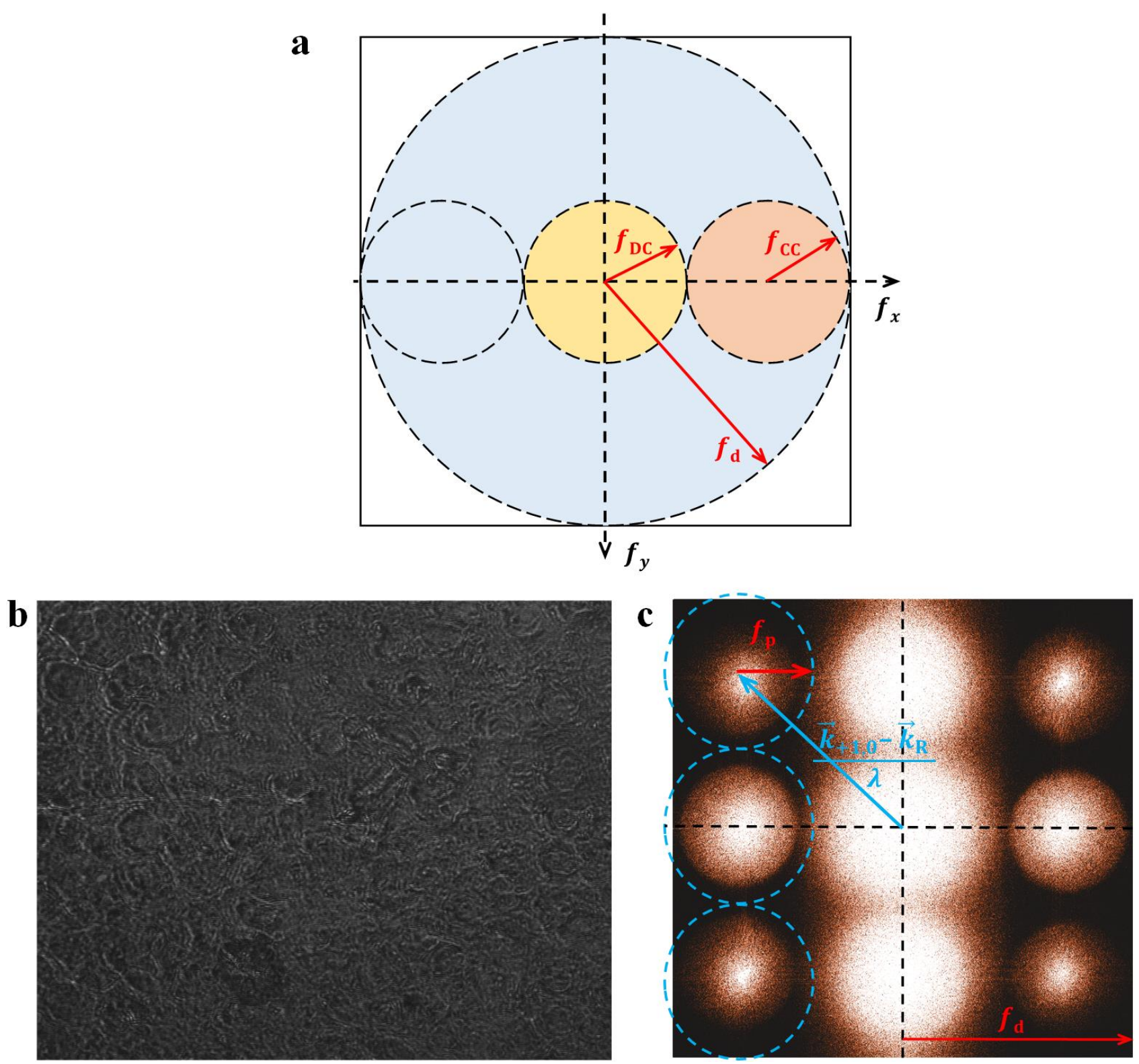

Figure S4: Quantifying the SPB of a spatial-bandwidth efficiency optimized $3 f$-MAIM. (a) The bandwidth limitations in the conventional DHM. $f_{\mathrm{DC}}$, the maximal frequency of the DC term. $f_{\mathrm{CC}}$, the maximum frequency of the cross-correlation (CC) terms. For the conventional off-axis DHM, when the object wave is weaker than the reference wave, it can be assumed that $f_{\mathrm{DC}}$ is equal to $f_{\mathrm{CC}}$. Thus, $f_{\mathrm{CC}}$ equals $\sim 1 / 3 \cdot f_{\mathrm{d}}$. (b) Interferogram of an unstained tissue slice obtained with the optimized $3 f$-MAIM and a $20 \times$ objective lens ( $0.4 \mathrm{NA}$ ), and (c) the corresponding Fourier spectrum. $f_{\mathrm{p}}$ equals $\sim 1 / 3 \cdot f_{\mathrm{d}}$. This optimization is achieved by employing a pair of Ronchi gratings of a higher frequency $(50 \mathrm{c} / \mathrm{mm})$ and decreasing the distance $d_{3}$. The optimization strategy can also be applied to 4/5f-MAIM. 


\section{Supplementary information}

As MAIM is a single-shot technique, its SBP is mostly limited by the pixel number of the detector. Specifically, for a given detector, the achieved SBP of MAIM can be illustrated by calculating the percentage of the occupied area in its Fourier space.

As both phase and amplitude channels contribute independently to the SBP in a coherent imaging system, the SPB of the conventional DHM can be quantified by:

$$
\mathrm{SBP}_{\mathrm{co}}=2\left(f_{\mathrm{Cd}} / f_{\mathrm{d}}\right)^{2} \cdot \mathrm{SBP}_{\mathrm{d}} \approx 2 / 9 \cdot \mathrm{SBP}_{\mathrm{d}},
$$

where $\mathrm{SBP}_{\mathrm{d}}$ is the maximum $\mathrm{SBP}$ that can be captured by the detector, which equals $1 / 4$ of its pixel number according to the Nyquist criterion.

Since the optimized 3f-MAIM provides 3 sub-FOVs (Figure S4 c), its SPB can be quantified by:

$$
\mathrm{SBP}_{3 f-\mathrm{MAIM}}=3 \cdot 2 \cdot\left(f_{\mathrm{p}} / f_{\mathrm{d}}\right)^{2} \cdot \mathrm{SBP}_{\mathrm{d}} \approx 2 / 3 \cdot \mathrm{SBP}_{\mathrm{d}} .
$$

Thus, the SBP of the optimized $3 f$-MAIM approaches $2 / 3$ of the SBP of bright-field microscopy $\left(\mathrm{SBP}_{\mathrm{d}}\right)$ and is about 3 times larger than that of the conventional DHM. 


\section{Supplementary information}

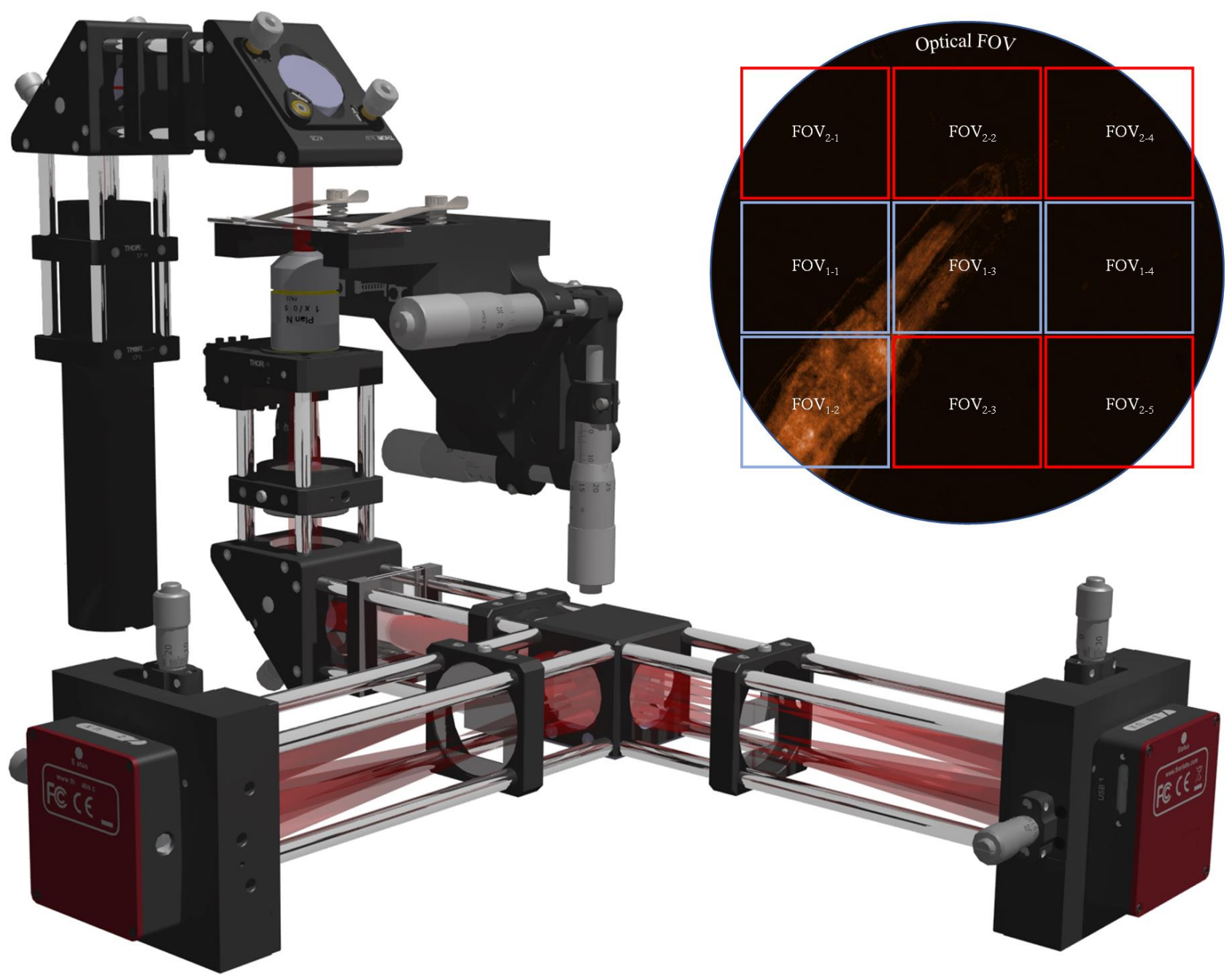

Figure S5: Schematic of a possible implementation of dual-channel MAIM. The complementary 4 and 5f-MAIM modules capture wavefront subsets that contain sample information without overlapping. In particular, the $4 f$ MAIM module captures the sample images from $\mathrm{FOV}_{1-1}-\mathrm{FOV}_{1-4}$, and the $5 f$-MAIM module covers $\mathrm{FOV}_{2-1}-\mathrm{FOV}_{2-}$ 5. By adopting a dual-triggering scheme, the dual-channel MAIM can achieve quasi single-shot quantitative phase imaging with a rectangle synthetic FOV and improve the SBP by a factor of 9. 


\section{Supplementary information}
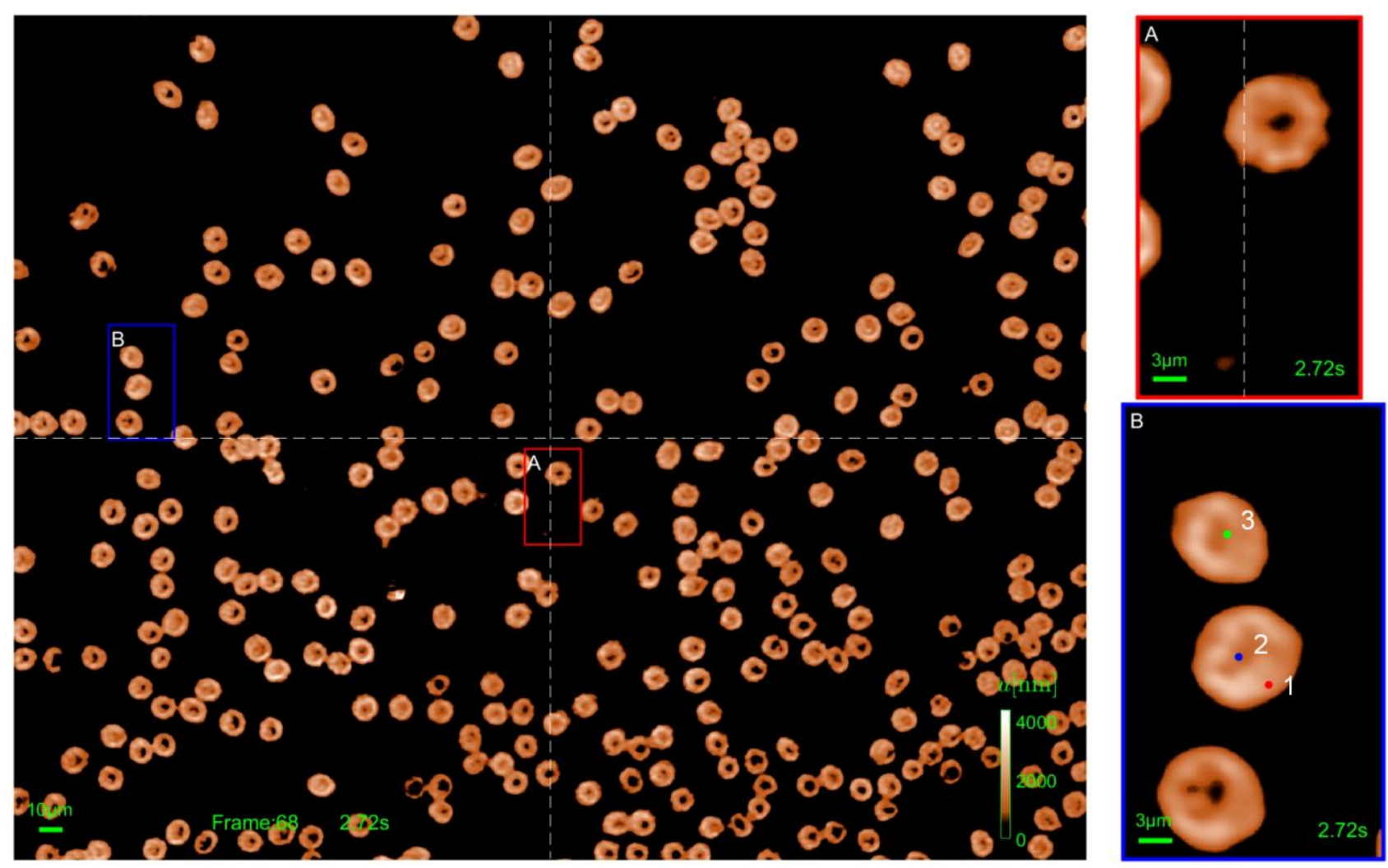

Figure S6: Brief description of Video S1. The additional dotted lines delimit the 4 FOV subsets (camera FOV). The zoomed-in video A and B are from the small box area A and B, respectively. A spans two FOV subsets, and B reveals nanoscale subcellular dynamics. 